\title{
TRIAXIALITY IN THE BULGES OF SPIRALS: DYNAMICAL IMPLICATIONS
}

\author{
A.M. VARELA ${ }^{1}$, E. SIMONNEAU ${ }^{2}$, C. MUÑOZ-TUÑÓN ${ }^{1}$
}

1. Instituto de Astrofísica de Canarias, 38200, La Laguna, Tenerife, Spain.

2. Institut d'Astrophysique de Paris, 98 bis, Boul. Arago, 75014 Paris, France.

The presence of triaxial bulges has been suggested as a mechanism for sweeping out the interstellar gas into the nuclear zones of normal spiral galaxies, giving rise to star formation processes (Zaritsky and Lo, 1986). In an initial phase of the present study, we have analysed the bulge component of a sample of spiral galaxies, obtained using the $4.2 \mathrm{~m}$ WHT at La Palma Observatory, selected from among those which exhibit moderate activity. The results obtained are: (a) There is evidence, from the misalignment between the semi-major axes of the isophotes of the bulge and disc components, to support the presence of triaxial bulges in these galaxies; (b) The high spatial resolution of the images allows us to sample the bulges, showing that the surface brightness distribution is not always represented by a family of homologous coaxial ellipses, but rather by a set of ellipses which precess around a common centre, and whose ellipticity varies (see Beckman et al., 1991; Varela, 1992). It is possible to reproduce this structure with a family of concentric triaxial ellipsoidal shells. The presence of a disc offers the advantage of enabling us to interpret the geometry of the bulge correctly, but poses the disadvantage of requiring to interpret the disc emission in order to reconstruct that associated only with the bulge, i.e. to decontaminate the bulge from the disc light. To this end we carried out a photometric analysis, which enabled us to effect a valid bulge/disc decomposition. The preliminary results yielded by the analysis of the bulge component (after decontaminating the disc) show the presence of "transparent" discs in the galaxies analysed.

The absence of axisymmetry in the inferred bulge potential, in the context of galactic rotation, might be responsible for driving the gas into the central region of the bulge, and must therefore be considered when accounting for the formation of rings, the production of gas flows, etc., and in the evolutionary model of starbursts. In order to analyse quadrupole moments and, more generally, the dynamics of these triaxial bulges, we must determine the spatial distribution of the bulges in the spirals analysed, as derived from the observed isophotes, assuming a mass to light ratio. In some cases, the spatial source and the surface brightness distributions are related by the Abel integral transform (Stark, 1977; Simonneau, Varela, Muñoz-Tuñón, 1992), but if differential precession exists between isophotes we do not, in general, obtain an integral relation, nor is it even possible to specify analytically the surface brightness contours. In these cases we adopt a direct method to synthesize photometrically the 3-D structure of the bulge. It is possible to reproduce this morphology with a family of 
ellipsoidal shells of equal source density which are concentric and coaxial, but with varying axial ratios. For each individual object under study, we intend to model a three-dimensional structure (see in bottom figure the spatial source distribution associated to different 3-D solutions for NGC 4736, corresponding to different axial ratios families) capable of reproducing the observations (pixel size is 27"). The distorsions detected could play a fundamental role in the dynamics of the circumnuclear gas (see Muñoz-Tuñón and Varela, 1991).

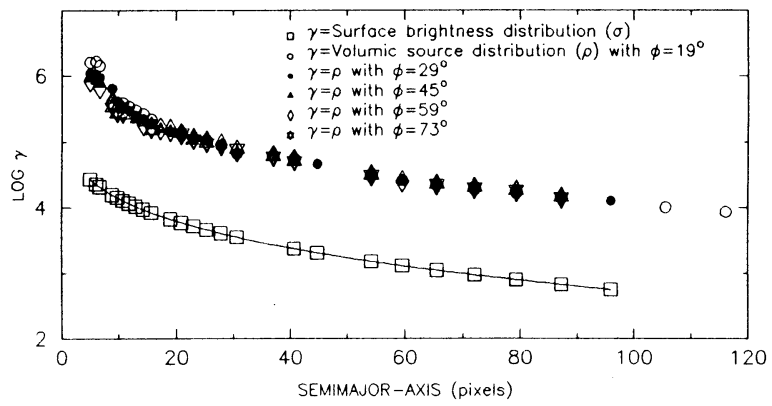

Conclusions

1. The surface brightness within the bulge is reproduced by a family of ellipses that precess around a common centre.

2. The spatial source distribution has been modelled and we have developed a spectral analytical method to inverse the 2-D structure.

3. It is possible to reproduce the surface brightness distribution with a family of concentric, coaxial ellipsoids with variable axial ratios.

4. In general, the concepts projection and integration along line of sight have different meanings, and one cannot be used in the context of the other.

5. Quadrupolar moments for the gravitational potential are present on the disc and are not negligible, depending on the azimuth.

6. The presence of a triaxial potential associated to the bulge component should be taken into account when modelling the stable inner annular structures. This is an alternative to the classical interpretation of explosive nuclear starburst.

\section{References}

Beckman, J.E.; Varela, A.M.; Muñoz-Tuñón, C.; Vílchez, J.M.; Cepa, J.: 1991, Astron. Astrophys., 245, 436.

Muñoz-Tuñón, C.; Varela, A.M.: 1991, Proc. Workshop Relationships between Active Galactic Nuclei and Starburst Galaxies, China (in press).

Simonneau, E.; Varela, A.M.; Muñoz-Tuñón, C.: 1992, JQSRT (in press).

Stark, A.A.: 1977, Astrophys. J., 213, 368.

Varela, A.M.: 1992, Ph.D. Thesis, Univ. La Laguna.

Zaritsky, D.; Lo, K.Y.: 1986, Astrophys. J., 303, 66. 